\title{
New onset rheumatoid arthritis with refractory hyper-eosinophilia associated with inactivated COVID-19 vaccine
}

\section{Rohit Singh}

Banaras Hindu University

Upinder Kaur ( $\nabla$ drupinder.bhu@gmail.com )

Banaras Hindu University

Ankur Singh

Ankuram Labs

Sankha Shubhra Chakrabarti ( $\nabla$ sankha.geriatrics@gmail.com )

Banaras Hindu University

\section{Case Report}

Keywords: rheumatoid, connective, autoimmune, nodule, eosinophilia

Posted Date: January 6th, 2022

DOI: https://doi.org/10.21203/rs.3.rs-1236248/v1

License: (c) (i) This work is licensed under a Creative Commons Attribution 4.0 International License. Read Full License 


\section{Abstract}

COVID-19 vaccines are considered one of the primary strategies for countering the pandemic. While mRNA based and viral vector-based vaccines have been predominantly used, inactivated SARS-CoV-2 vaccines are being manufactured in countries such as China and India. Post approval, rare but serious adverse events such as myocarditis and stroke have been observed with mRNA based and viral vectored COVID-19 vaccines. Inactivated vaccines in general have shown better tolerability in clinical trials. Here we report the first case of new-onset seropositive rheumatoid arthritis (RA) with rheumatoid nodules and refractory reactive eosinophilia within two weeks of receiving an inactivated COVID-19 vaccine (COVAXIN).

\section{Introduction}

COVID-19 vaccines are considered one of the primary strategies for countering the pandemic. While mRNA based and viral vector-based vaccines have been predominantly used, inactivated SARS-CoV-2 vaccines are being manufactured in countries such as China and India. Post approval, rare but serious adverse events such as myocarditis and stroke have been observed with mRNA based and viral vectored COVID-19 vaccines. ${ }^{1,2}$ Inactivated vaccines in general have shown better tolerability in clinical trials. Here we report the first case of new-onset seropositive rheumatoid arthritis (RA) with rheumatoid nodules and refractory reactive eosinophilia within two weeks of receiving an inactivated COVID-19 vaccine (COVAXIN).

\section{Case Report}

An elderly female in the late 50s with a medical history of hypertension, well controlled on amlodipine and hydrochlorothiazide therapy, developed acute onset facial puffiness, dysphagia, tongue, and neck swelling, with an onset one week after the first dose of COVAXIN. Leucocytosis with eosinophilia in her general blood picture had prompted referral to our centre. She had no personal or family history of an autoimmune or allergic disorder. Swelling was observed on her face, tongue, and neck. Rest of the physical examination was non-contributory. Further workup was performed focusing on rising eosinophil counts $(>30 \%)$. Her serum total IgE was high. Stool examination was negative for parasitic ova, cysts, or atypical organisms. Though no cardiac symptoms were present, the B-type natriuretic peptide levels were elevated nearly four times of the upper limit of normal. An upper gastrointestinal endoscopy and biopsy performed as part of evaluation for dysphagia revealed no abnormalities, neither did a cardiac magnetic resonance imaging. The patient was started empirically on oral prednisolone at a low dose of $10 \mathrm{mg}$ once daily, considering a provisional diagnosis of vaccine induced eosinophilia with asymptomatic myocarditis. Her initial complaints improved over the next one month, but she developed a few bony nodules near both her elbow joints. This was followed by severe pain and swelling of bilateral elbow, wrist, metacarpophalangeal (MCP) and proximal interphalangeal (PIP) joints, sparing the distal 
interphalangeal joints (DIP). Serology for collagen vascular diseases was performed, and a high titre of rheumatoid factor (RA factor) and anti-cyclic citrullinated peptide (anti-CCP) were observed. Other auto antibodies including anti-nuclear antibody were not detected. The patient's eosinophilia persisted rising up to $55 \%$ on differential count. A bone marrow biopsy was performed which revealed a hypercellular marrow with $90 \%$ morphologically normal eosinophils (Figure 1). Biopsy of the elbow nodules showed palisading granulomas with cores of necrobiotic collagen, consistent with rheumatoid nodule. The patient was diagnosed as a case of new onset rheumatoid arthritis following inactivated COVID-19 vaccine, and was started on weekly methotrexate $7.5 \mathrm{mg}$, and daily prednisolone $20 \mathrm{mg}$ and indomethacin $75 \mathrm{mg}$ for pain control. Initial response in pain was significant, and the patient is on followup.

\section{Discussion}

Patients with autoimmune diseases were largely excluded from the controlled trials of COVID-19 vaccines. As such, the interaction of vaccines with the underlying autoinflammatory state has been observed in few real-world studies. ${ }^{3,4}$ Post approval, rare but serious adverse events including vaccine induced thrombosis and thrombocytopenia (VITT) and myocarditis have been reported with adenoviral vectored and mRNA based COVID-19 vaccines. ${ }^{1,2,5}$ The inactivated vaccines in general have displayed a favourable safety profile in controlled settings. In rare circumstances, however, cardiac, and new onset musculoskeletal phenomena have been reported. We previously reported a case of hyper-eosinophilia with symptomatic myocarditis, facial, hand and ankle edema in a medical professional within days of his first dose of COVAXIN. ${ }^{6}$ New onset reactive arthritis has been observed in elderly patients after administration of the inactivated vaccine, CoronaVac. ${ }^{7}$ Flares of systemic rheumatic disease have been observed in around $15 \%$ of recipients of COVID-19 vaccines, largely the mRNA vaccines. Majority of such flares have been reported to be of moderate-severe category with some persisting for more than 3 weeks. ${ }^{3}$ To our knowledge, this is the first case of new onset rheumatoid arthritis with characteristic rheumatoid nodules post COVID-19 vaccine. The associated hyper eosinophilia was refractory to steroid therapy. Though the patient had elevated BNP levels, there were no symptoms or signs of cardiac involvement. However, subclinical myocarditis cannot be ruled out. The lady had a background history of controlled hypertension. Previously, women and those with pre-existing hypertension each have been shown to be associated with a two-times higher odds of development of adverse events following immunization. ${ }^{8}$ The mechanisms of autoimmune induction by inactivated COVID-19 vaccine needs to be delineated. It can be related to the aluminium hydroxide adjuvant present in the vaccine. Autoimmune/inflammatory syndromes induced by adjuvant (ASIA) was first described in 2011 by Shoenfeld and Agmon-Levin. Aluminium in adjuvants is known to cause preferential Th2 based IL-5 stimulation that can explain hyper eosinophilia and autoantibodies seen in the patient. The musculoskeletal inflammation can also be explained by the NOD-like receptor protein family 3 (NLRP3) driven release of IL-1 and IL-18 by aluminium. Persons with certain HLA alleles such as HLA-DRB1 might be at increased risk. ${ }^{9}$ Autoantibodies generated as a response to viral proteins due to cross-reactivity, and direct toxicity by the Spike or other viral proteins, may be other tentative pathogenetic pathways. ${ }^{10}$ 
The case is interesting as the first reported case of a new onset connective tissue disorder following inactivated COVID-19 vaccine administration. The rapidity of disease progression including appearance of rheumatoid nodules is another atypical feature of this case. Focused research is needed on autoimmune complications resulting from COVID-19 vaccines, and there is also a constant need to update guidelines for vaccinating patients with autoimmune conditions using diverse vaccine types, including the updating of potential contraindications.

\section{Declarations}

Acknowledgement: None

Conflicts of Interest: None

Funding support: None

Ethical Statement: No human experimentation performed. Individual case reports do not require ethical committee permission. All procedures performed following the Declaration of Helsinki and its modifications. Written informed consent taken from the patient's legal guardian for publication of the case.

\section{References}

1. Montgomery J, Ryan M, Engler R, et al. Myocarditis Following Immunization With mRNA COVID-19 Vaccines in Members of the US Military. JAMA Cardio/ 2021; published online June 29.

DOI:10.1001/jamacardio.2021.2833.

2. Schultz NH, Sørvoll IH, Michelsen AE, et al. Thrombosis and Thrombocytopenia after ChAdOx1 nCoV19 Vaccination. N Engl J Med 2021; 384: 2124-30.

3. Barbhaiya M, Levine JM, Bykerk VP, Jannat-Khah D, MandI LA. Systemic rheumatic disease flares after SARS-CoV-2 vaccination among rheumatology outpatients in New York City. Ann Rheum Dis 2021; 80: 1352-4.

4. Sattui SE, Liew JW, Kennedy K, et al. Early experience of COVID-19 vaccination in adults with systemic rheumatic diseases: results from the COVID-19 Global Rheumatology Alliance Vaccine Survey. RMD Open 2021; 7: e001814.

5. Singh, Rohit; Chakrabarti, Sankha Shubhra; Gambhir IS et al. Acute cardiac events following ChAdOx1 nCoV-19 corona virus vaccine: report of three cases. Res Sq 2021;: 4-10.

6. Tiwari, Ashutosh; Karna $\mathrm{G}$ et al. Hyper-eosinophilic syndrome with myocarditis after inactivated SARS-CoV-2 vaccination: A case study. Res Sq 2021;: 4-9.

7. Türk SM, Öztürk Z, Karataş D, Gönüllü E. INACTIVATED COVID-19 VACCINE CAN INDUCE REACTIVE POLYARTHRITIS IN OLDER PATIENTS: REPORT OF TWO CASES. Georgian Med News 2021;: 100-2. 
8. Kaur U, Ojha B, Pathak BK, et al. A prospective observational safety study on ChAdOx1 nCoV-19 corona virus vaccine (recombinant) use in healthcare workers- first results from India. EClinicalMedicine 2021;: 101038.

9. Shoenfeld Y, Agmon-Levin N. 'ASIA' - Autoimmune/inflammatory syndrome induced by adjuvants. $J$ Autoimmun 2011; 36: 4-8.

10. Vojdani A, Vojdani E, Kharrazian D. Reaction of Human Monoclonal Antibodies to SARS-CoV-2 Proteins With Tissue Antigens: Implications for Autoimmune Diseases. Front Immuno/ 2021; 11. DOI:10.3389/fimmu.2020.617089.

\section{Figures}

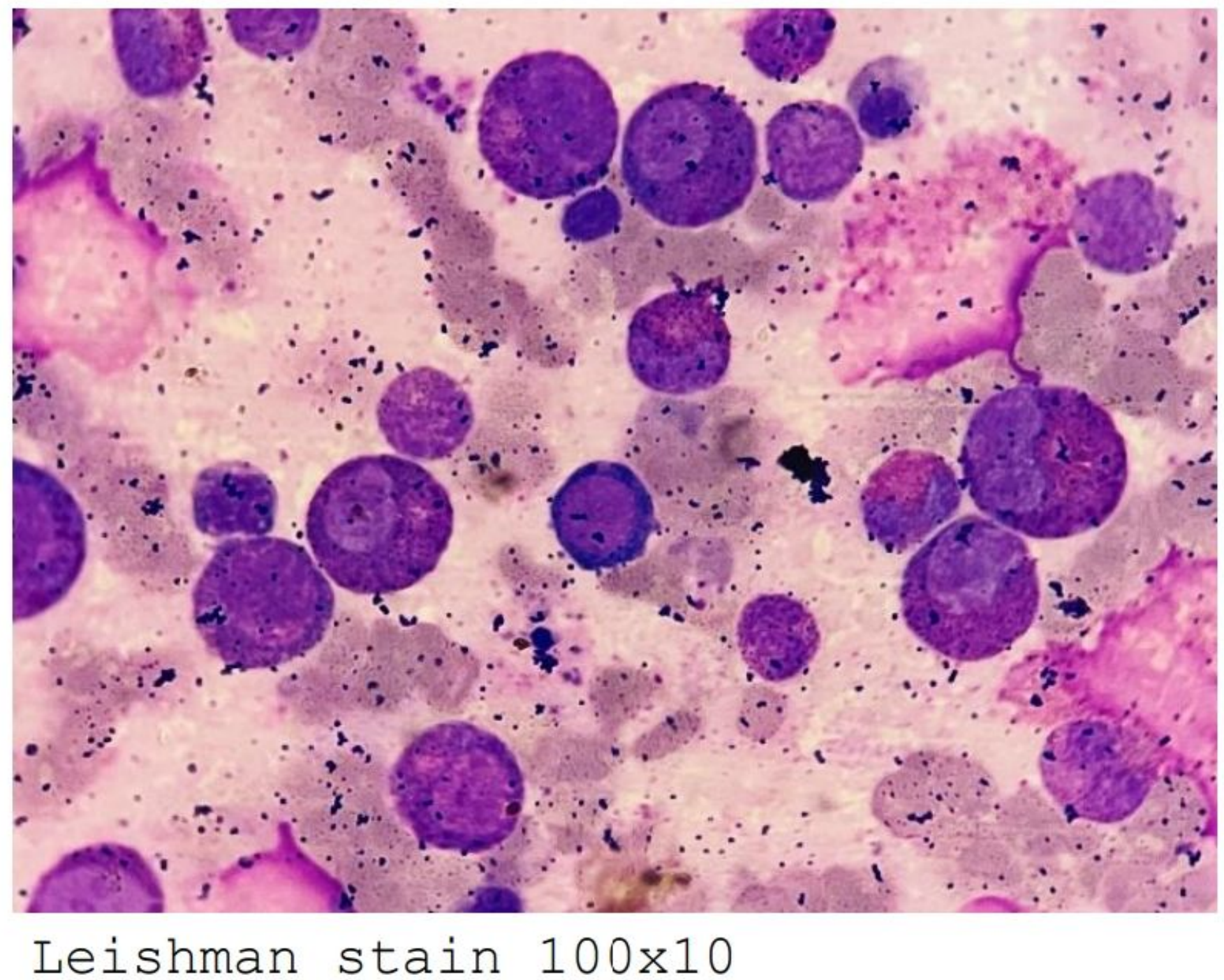

Figure 1 
Legend not included with this version.

Page 6/6 